A N N A L E S Annales de Bretagne et des Pays de l'Ouest

\title{
La naissance de la bibliothèque municipale de
} Rennes 1789-1803

The birth of the Rennes municipal library 1789-1803

\section{Morgane Egea}

\section{OpenEdition}

\section{Journals}

Édition électronique

URL : http://journals.openedition.org/abpo/2006

DOI : $10.4000 / a b p o .2006$

ISSN : 2108-6443

Éditeur

Presses universitaires de Rennes

\section{Édition imprimée}

Date de publication : 30 mai 2011

ISBN : 978-2-7535-1691-5

ISSN : 0399-0826

\section{Référence électronique}

Morgane Egea, "La naissance de la bibliothèque municipale de Rennes 1789-1803 », Annales de

Bretagne et des Pays de l'Ouest [En ligne], 118-2 | 2011, mis en ligne le 23 juin 2013, consulté le 22 avril 2019. URL : http://journals.openedition.org/abpo/2006 ; DOI : 10.4000/abpo.2006 


\title{
La naissance de la bibliothèque municipale de Rennes 1789-1803 ${ }^{1}$
}

\author{
Morgane EGEA \\ Étudiante en master 2 \\ université Rennes 2 Haute-Bretagne
}

À la fin de l'Ancien Régime, Rennes, capitale provinciale, est une ville universitaire. De la présence du parlement de Bretagne, elle tire une renommée qui la distingue par rapport aux autres villes de la France de l'ouest. Ce sont les robins qui constituent alors son élite intellectuelle. Pour eux, le livre n'est pas seulement un instrument de travail, il est aussi un outil de culture primordial. Liés aux formes anciennes, traditionnelles et savantes de la culture, ils sont sensibles à son prestige ${ }^{2}$. Ainsi contribuent-ils à la diffusion du livre à Rennes, et, ce faisant, à façonner l'identité culturelle de la ville. Rennes compte d'ailleurs de grandes bibliothèques parlementaires à l'époque, par exemple celle du président de Robien ${ }^{3}$. Certains ecclésiastiques aussi possèdent des bibliothèques, principalement composées de livres religieux. Les Rennais du XVIII ${ }^{\mathrm{e}}$ siècle peuvent se procurer des livres auprès des libraires ${ }^{4}$ ou des revendeurs de livres d'occasion ${ }^{5}$. Mais ils ont également accès à la lecture par l'intermédiaire des sociétés de pensée ${ }^{6}$,

1. Cet article est tiré, en grande partie, des deux premiers chapitres du mémoire de master 2 (La naissance de la bibliothèque municipale de Rennes : 1793-1818) soutenu en 2008 à l'université Rennes 2. Je tiens à remercier Gauthier Aubert pour avoir dirigé ce travail de master, ainsi que les membres du jury de soutenance, Annie Antoine, professeur d'histoire moderne à l'université Rennes 2 et Marine Bedel, conservatrice à la bibliothèque des Champs Libres et de la ville de Rennes.

2. QuÉNIART, Jean, Culture et société urbaines dans la France de l'Ouest au XVIIT siècle, Paris, Klincksieck, 1978, p. 518-519.

3. AUBERT, Gauthier, Le Président de Robien, gentilhomme et savant dans la Bretagne des Lumières, Rennes, PUR, 2001.

4. SOREL, Patricia, La Révolution du livre et de la presse en Bretagne (1780-1830), Rennes, PUR, 2004.

5. JOUON DES LONGRAIS, Frédéric, " Le commerce des vieux livres à Rennes au XVIII ${ }^{\mathrm{e}}$ siècle ", Bulletin et Mémoires de la Société archéologique du département d'Ille-et-Vilaine, t. 37, 1907, p. 193-206.

6. VILLERS, Louis (de), "Les sociétés littéraires et scientifiques en Bretagne au $\mathrm{XVIII}^{\mathrm{e}}$ siècle "Bulletin et Mémoires de la Société archéologique du département d'Ille-et- 
grâce aux bibliothèques des communautés religieuses qui ouvrent leurs portes au public - ainsi celles des Carmes, des Bénédictins, des Jacobins et des Capucins ${ }^{7}$ - ou bien grâce à la bibliothèque des avocats ${ }^{8}$, elle aussi ouverte au public à la fin du siècle.

Rappelons que l'idée de bibliothèque publique se développe dans tout le pays à la fin de l'Ancien Régime, notamment grâce aux Lumières, qui, pardelà leur diversité, entreprennent de promouvoir un idéal démocratique et égalitaire articulé au désir d'utilité ${ }^{9}$. Si la nation entend alors s'approprier son patrimoine, c'est pour garantir l'instruction du peuple et régénérer la culture. La Révolution, par sa politique de confiscations, permet précisément aux projets des Lumières de devenir réalité car les biens nationaux deviennent dès lors la propriété de tous et rendent ainsi possible la création d'établissements culturels tels que les bibliothèques, qui, parce qu'elles sont libres d'accès, répondent à cette conception démocratique de la culture ${ }^{10}$. La question de la genèse des bibliothèques municipales de province sous la Révolution demeure pourtant méconnue à l'heure qu'il est. Les études effectuées sur ce sujet sont dispersées, peu visibles ou difficilement accessibles. Quelques articles ont été écrits, notamment à l'occasion du bicentenaire de la Révolution française ${ }^{11}$. On trouve également des informations sur l'origine des bibliothèques municipales dans l'Histoire des bibliothèques françaises ${ }^{12}$, ouvrage faisant la synthèse des connaissances acquises jusque-là. Les notices contenues dans les dix volumes de Patrimoine des bibliothèques de France. Un guide des régions ${ }^{13}$, qui témoignent de la richesse des fonds conservés aujourd'hui dans les bibliothèques françaises, reviennent aussi en quelques lignes sur l'histoire des collections ayant pour origine les confiscations révolutionnaires. Autant de travaux qui évoquent généralement, plus ou moins rapidement, les contraintes matérielles rencontrées à cette époque et indissociables de l'histoire de ces bibliothèques.

Notre recherche met spécifiquement l'accent sur ces questions d'ordre matériel afin de décrire de façon complète la naissance de la bibliothèque

Vilaine, t. 40, 1910, p. 193-235. BARRE, Catherine, La Chambre de lecture de Rennes : 17751875, maîtrise d'Histoire, université Rennes 2, 1972. KERJAN, Daniel, Rennes : les francsmaçons du Grand Orient de France. 1748-1998 : 250 ans dans la ville, Rennes, PUR, 2005.

7. GURY, Jacques, " Lire à Rennes de Louis XVI à Louis-Philippe ", Mémoires de la Société d'histoire d'archéologie de Bretagne, t. 62, 1985, p. 399.

8. RaISON, Estelle, L'Ordre des avocats rennais et sa bibliothèque au XVIII siècle, master d'Histoire, université Rennes 2, 2007.

9. RIOUX, Jean-Pierre et SiRINELLI Jean-François (dir.), Histoire culturelle de la France, vol. 3 : Lumières et liberté, les dix-huitième et dix-neuvième siècles, Paris, Seuil, 2005, p. 150.

10. Poulot, Dominique, Patrimoine et musées : l'institution de la culture, Paris, Hachette, 2001, p. 50.

11. Bulletin des Bibliothèques de France, 1989/2-3, Dossier 1789-1989 [http://bbf.enssib.fr].

12. VARRY, Dominique (dir.), Histoire des bibliothèques françaises : les bibliothèques de la Révolution et du XIX siècle, 1789-1914, Paris, Promodis-Éd. du Cercle de la Librairie, 1991.

13. Patrimoine des bibliothèques de France. Un guide des régions, Paris, Payot, 10 vol., 1995. 
municipale de Rennes pendant la Révolution et de ne négliger aucun aspect de son histoire ${ }^{14}$. Car, nous allons le voir, la réalisation de l'idéal révolutionnaire est chose difficile; les réformes mises en œuvre au cours de cette décennie fondatrice se heurtent à de multiples contraintes : non seulement l'organisation des bibliothèques est lente en raison des différents décrets, lois et instructions qui se succèdent, mais elle doit compter avec d'importantes difficultés matérielles, comme c'est le cas à Rennes. Ainsi verrons-nous d'abord comment des bibliothèques privées rennaises ont été saisies lors des confiscations révolutionnaires, puis comment elles ont été regroupées dans des dépôts littéraires en vue de constituer une bibliothèque publique, enfin comment les collections qui en sont issues ont dû passer par de nombreux déménagements avant de donner naissance à la bibliothèque municipale de Rennes.

\section{Les confiscations révolutionnaires}

\section{Trois vagues de confiscations}

L'année 1789 constitue une rupture essentielle dans l'histoire politique et sociale de la France, mais également dans celle de ses bibliothèques. Avant la Révolution, les bibliothèques étaient essentiellement des collections privées, appartenant à des institutions religieuses, à des particuliers ou bien à des académies et sociétés savantes. Certaines de ces bibliothèques étaient ouvertes au public, même si en réalité, seule une minorité d'érudits y avait vraiment accès ${ }^{15}$. Les décisions prises à partir de 1789 anéantissent l'organisation de ces bibliothèques d'Ancien Régime.

La première vague de confiscations a lieu en 1789. Cette année-là, les États généraux se réunissent pour faire face à la crise financière qui frappe l'État. La solution envisagée par l'Assemblée constituante est la nationalisation des biens du clergé. Elle est rapidement mise en œuvre : le décret du 2 novembre 1789 met les biens du clergé " à la disposition de la Nation ". La deuxième vague de confiscations débute avec le décret du 9 février

14. Notre travail, notamment la partie consacrée aux inventaires des confiscations, doit beaucoup au livre de Dominique VARRY, Sous la main de la Nation : les bibliothèques de l'Eure confisquées sous la Révolution Française, Ferney-Voltaire, Centre international d'étude du XVIII ${ }^{\mathrm{e}}$ siècle, 2005. Dans cette étude provinciale qui traite des saisies révolutionnaires dans le département de l'Eure, l'auteur s'attache à l'aspect matériel, par exemple lorsqu'il décrit la façon dont les inventaires sont réalisés ou lorsqu'il étudie l'emplacement des livres chez les victimes des confiscations. Cependant, cet ouvrage fournit uniquement un état des lieux de ce qu'étaient les bibliothèques privées des élites de la fin de l'Ancien Régime mais il ne constitue pas une histoire de l'émergence des bibliothèques publiques.

15. En 1789, il existe en France une cinquantaine de bibliothèques publiques. Ce terme de " bibliothèque publique " n'a pas sous l'Ancien Régime le sens que nous lui attribuons actuellement : c'est alors avant tout, par opposition aux collections strictement privées, une bibliothèque accessible au public, qui ne relève pas nécessairement d'une autorité de droit public (Jolly, Claude [dir.], Histoire des bibliothèques françaises : les bibliothèques sous l'Ancien Régime, 1530-1789, Paris, Promodis-Éd. du Cercle de la Librairie, 1988, p. 391). 
1792, qui ordonne la saisie des biens des émigrés, considérés comme des ennemis de la Révolution. Ces saisies concernent également les biens des ecclésiastiques déportés. Enfin, la troisième vague de confiscations a lieu en 1793. Elle est consécutive à la suppression des universités, des sociétés littéraires, des académies et des corporations. Le 8 août 1793, leurs bibliothèques sont confisquées ${ }^{16}$.

Ces mesures, décidées au niveau national, sont appliquées à Rennes. Sont tout d'abord saisies treize bibliothèques provenant des établissements religieux rennais supprimés : sept d'hommes (les grands Carmes, les Carmes, les Capucins, les Minimes, les Augustins, les Bénédictins, les Jacobins) et cinq de femmes (les religieuses du Calvaire, du Colombier, les Carmélites, les dames Budes et la bibliothèque de l'abbesse de SaintGeorges). Il faut ajouter à ces bibliothèques celle du petit séminaire. Vingtneuf bibliothèques d'ecclésiastiques séculiers sont également saisies, ainsi que celles d'une vingtaine d'émigrés, celle de l'école de droit et enfin la bibliothèque des avocats. Au total, ce sont soixante-huit bibliothèques plus ou moins importantes qui ont été confisquées à Rennes. Certaines de ces bibliothèques sont regroupées et constituent un ensemble de quarante-cinq dépôts ${ }^{17}$ destiné à former la bibliothèque publique de Rennes.

\section{La réalisation des inventaires}

Soucieux d'organiser le fonctionnement des bibliothèques, l'État montre très rapidement sa volonté de connaître les richesses bibliographiques dont il devient dépositaire. Cette volonté de répertorier l'état des collections confisquées se traduit par la réalisation de nombreux inventaires entre 1790 et 1794. Ceux-ci décrivent pièce par pièce les maisons visitées. Mis à part les communautés religieuses, qui possèdent en général une bibliothèque commune, les livres sont le plus souvent répartis en de multiples pièces d'une même maison. La chambre, ainsi que le cabinet, sont les lieux privilégiés de leur rangement. Ils sont conservés dans un meuble servant de bibliothèque, la plupart du temps dans une simple armoire, qui peut permettre de classer les volumes par formats sur les rayons. Ainsi, chez Fournier du Trélo, l'inventaire mentionne tout d'abord " dans la chambre à côté servant de cabinet, une bibliothèque composée de livres de divers formats ", puis "dans une seconde chambre une armoire de bibliothèque où il y a peu de livres ", enfin " dans le vestibule une armoire d'attache remplie de livres ${ }^{18} "$.

La méthode employée pour la rédaction des inventaires est toujours la même. Dans les communautés religieuses, ils sont dressés avant la

16. ROBERT, Ulysse, Recueil des lois, décrets, ordonnances, concernant les bibliothèques publiques, Paris, H. Champion, 1883.

17. Bibliothèque municipale de Rennes, Ms 562 - Catalogue des dépôts littéraires.

18. Arch. dép. d'Ille-et-Vilaine, 1 Q 972 - Biens de deuxième origine : émigrés, détenus, suspects. Dossier individuel : Fournier du Trélo. 
dispersion des religieux et en présence des supérieurs. Chez les particuliers, en revanche, ils le sont alors que ces derniers ont déjà quitté les lieux. Les inventaires sont généralement effectués par deux commissaires, accompagnés d'un greffier. Les commissaires sont des officiers municipaux, nommés par le directoire du district. Ils font partie de l'élite de la ville ${ }^{19}$, mais ils ne possèdent pas forcément les compétences nécessaires pour inventorier les livres, estimer l'intérêt et la valeur des ouvrages. Des libraires sont donc parfois employés pour les aider. C'est ainsi que, le 19 octobre 1791, le libraire René Etienne Courné est désigné par le directoire du district pour effectuer l'inventaire des bibliothèques confisquées ${ }^{20}$. Il se charge notamment de l'inventaire de la bibliothèque des Carmes déchaussés en $1792^{21}$. Il n'est pas le seul dans ce cas. Toujours en 1792, Orry contribue à l'inventaire de la maison de Cintré : les commissaires, après avoir réuni les livres dispersés et les avoir triés, avaient annoncé leur intention de faire appel à un libraire ${ }^{22}$. Enfin, le libraire Hamelin participe lui aussi aux inventaires. En 1794, il répertorie les livres de l'abbé Carron et consacre cinq jours à l'estimation de ceux de Saturnin du Bourblanc, qui représentent environ un millier de volumes ${ }^{23}$. Trois libraires rennais sont donc mentionnés dans les inventaires du mobilier confisqué, qui, grâce à leurs connaissances bibliographiques, ont contribué au travail d'inventaire des commissaires.

Les officiers municipaux rencontrent parfois des obstacles pour réaliser ces inventaires. Il arrive que la famille du propriétaire des biens qui doivent être inventoriés s'oppose à la visite domiciliaire, nie l'émigration dudit propriétaire, ou bien cache certains objets ${ }^{24}$. De même, le divorce est parfois utilisé par les femmes d'émigrés pour tenter de sauver la fortune de leur mari ${ }^{25}$. Il semble que ce soit le cas de Jeanne Duboys, divorcée de Fermond, qui réclame le partage des biens en 1794 et qui, par ce moyen,

19. À Rennes, une dizaine de commissaires différents ont réalisé ces inventaires, dont : Jean Corentin Louis Le Baron, avocat au parlement avant la Révolution; Sevestre, député d'Ille-et-Vilaine à la Convention nationale, greffier au tribunal et chef du club local des Jacobins en 1792; Charles Bonaventure Marie Toullier, avocat au parlement et agrégé de droit avant 1789 , puis membre du directoire du district; Jourdain, procureur au présidial avant la Révolution et Anger, avocat au parlement, membre du directoire du district en 1792 (Morabito, Marcel [dir.], La Révolution et les juristes à Rennes, Paris, Economica, 1989).

20. Arch. dép. d'Ille-et-Vilaine, 1 Q 662 - Séquestre du mobilier : bibliothèques : correspondance.

21. Arch. dép. d'Ille-et-Vilaine, 1 Q 663 - Catalogue de la bibliothèque des Carmes déchaussés.

22. Arch. dép. d'llle-et-Vilaine, 1 Q 994 - Biens de deuxième origine : émigrés, détenus, suspects. Dossier individuel Cintré.

23. Arch. dép. d'Ille-et-Vilaine, 1 Q 1090 - Biens de deuxième origine : membres du clergé. Dossier individuel Carron; et 1 Q 950 - Biens de deuxième origine : émigrés... Dossier individuel Bourblanc.

24. VARRY, Dominique, Sous la main de la Nation, op. cit., p. 33.

25. VARRY, Dominique (dir.), Histoire des bibliothèques françaises : les bibliothèques de la Révolution et du XIX siècle..., op. cit., p. 12. 
récupère entre autres la moitié des livres qui avaient été saisis ${ }^{26}$. De nombreux livres ont donc probablement échappé aux confiscations de ces différentes façons.

\section{Les inventaires : lacunes et partialité}

Les livres ne font pas l'objet d'un inventaire particulier et sont décrits comme le reste du mobilier. Les commissaires ne s'intéressant généralement pas plus aux livres qu'au mobilier, ils les énumèrent rarement un par un. Par exemple, l'inventaire des Bénédictins indique que « le surplus des ouvrages n'est plus qu'un amas informe de volumes dépareillés, bouquins de nulle valeur $^{27}$ ". Chez les particuliers, ces approximations sont également visibles. En témoigne cet inventaire, qui reste extrêmement flou en ce qui concerne les livres : " Une bibliothèque dans laquelle il y a différents volumes ${ }^{28}$. " Il existe sans doute quelques inventaires précieux, d'une grande précision, qui comportent quelquefois la liste de tous les livres, mentionnant leur titre, leur auteur, leur format et parfois la valeur à laquelle ils sont estimés. Mais leur existence tient généralement au fait que ce sont des libraires qui les ont dressés. La bibliothèque des Carmes déchaussés, par exemple, a été répertoriée par le libraire Courné, qui a réalisé un catalogue par ordre alphabétique d'auteurs ${ }^{29}$. La plupart du temps, les bibliothèques confisquées ne bénéficient en revanche que d'une description globale, quand elles ne sont pas signalées par un simple mot. Précisons enfin que de nombreux inventaires du mobilier ne comportent aucune mention de livre, mais que cela n'autorise pas à conclure à l'absence effective d'ouvrage dans la mesure où certaines bibliothèques se retrouvent dans les dépôts littéraires alors même qu'elles n'ont fait l'objet d'aucun inventaire lors des confiscations.

Il faut par ailleurs prendre en compte la part de subjectivité qui préside à l'élaboration de ces inventaires. Ainsi les livres usagés, qui sont sans doute les plus lus, ne sont que rarement décrits. Les commissaires se contentent de les désigner comme des "vieux bouquins" ou des "livres de nulle valeur ". De même, les commissaires se contentent très souvent de citer un ou deux titres jugés significatifs et délaissent les autres ouvrages, qui ne semblent pas mériter de description. L'exemple de cet inventaire est assez représentatif de cette pratique :

"Dans la salle suivante, un bas de bibliothèque rempli de volumes tant reliés que brochés in-quarto in-folio et in-12, parmi lesquels nous avons remarqué principalement sept volumes du dictionnaire des sciences, le dictionnaire historique, le dictionnaire de l'académie, le dictionnaire écono-

26. Arch. dép. d'Ille-et-Vilaine, 1 Q 969 - Biens de deuxième origine : émigrés... Dossier individuel Fermond.

27. Arch. dép. d'llle-et-Vilaine, 1 Q 853 -Biens de première origine : clergé régulier : Bénédictins.

28. Arch. dép. d'Ille-et-Vilaine, 1 Q 1102 - Biens de deuxième origine : membres du clergé. Dossier individuel Le Berneur.

29. Arch. dép. d'Ille-et-Vilaine, 1 Q 663 - Catalogue de la bibliothèque des Carmes déchaussés. 
mique, les éphémérides du citoyen, les autres ouvrages nous ont paru pour la plupart de jurisprudence ${ }^{30}$."

Du fait qu'elles risquent de les occuper longuement, les masses importantes de livres découragent tout particulièrement les commissaires. Ainsi, pour l'inventaire du petit séminaire, ceux-ci écrivent :

«Entrés ensuite dans la bibliothèque, considérant qu'elle renferme environ 12 ou 15000 volumes et que l'inventaire en seroit long [...] nous avons pris le parti de mettre le scellé sur les portes de la dite bibliothèque ${ }^{31}$. "

À l'inverse, lorsqu'un catalogue de bibliothèque existe déjà, le travail des commissaires se trouve évidemment facilité. Ils l'annexent alors au procès-verbal, après vérification. A titre d'exemple, ils procèdent de cette façon pour la bibliothèque des Augustins ${ }^{32}$.

La réalisation des inventaires peut être enfin compliquée par l'état des bibliothèques. Le travail des commissaires est notamment entravé par le désordre qui règne en plusieurs endroits. Ainsi la bibliothèque des Bénédictins " est réellement dans un état de confusion et de malpropreté [...] les livres y sont jettés pêle-mêle sur le plancher et les rayons de la bibliothèque n'en peuvent contenir davantage ${ }^{33}$ ". S'agissant de la bibliothèque des Cordeliers, les commissaires constatent :

"Vu le très grand nombre de volumes de différents formats particulièrement des in-folio et in-quarto la plupart dépareillés et le tout dans un tel désordre que nos autres fonctions publiques ne nous laissent pas un temps suffisant pour continuer l'inventaire vu surtout que nous n'avons trouvé aucun catalogue pour nous faciliter le travail ${ }^{34}$."

Enfin, chez les Capucins, les commissaires indiquent :

"Au second étage est la bibliothèque, mais quelque recherche que nous ayons faite tant de la clef d'ouverture, que de celle de la chambre du supérieur où l'un des domestiques nous a dit que pourroit se trouver la clef de la bibliothèque, nous n'avons trouvé ni l'une ni l'autre ${ }^{35}$. "

\section{Le sort incertain des livres confisqués}

Une fois les inventaires terminés, les bâtiments confisqués sont vendus progressivement. À Rennes, au fur et à mesure de ces ventes, les bibliothèques sont regroupées dans des dépôts provisoires ${ }^{36}$. Il est difficile d'évaluer l'ampleur des saisies. En effet, non seulement les inventaires ne recen-

30. Arch. dép. d'llle-et-Vilaine, 1 Q 1041 - Biens de deuxième origine : émigrés... Dossier individuel Moëlien.

31. Arch. dép. d'Ille-et-Vilaine, 1 Q 804 - Biens de première origine : clergé séculier : petit séminaire.

32. Arch. dép. d'llle-et-Vilaine, 1 Q 852 - Biens de première origine : clergé régulier : Augustins. 33. Arch. dép. d'llle-et-Vilaine, 1 Q 853 - Biens de première origine : clergé régulier : Bénédictins. 34. Arch. dép. d'Ille-et-Vilaine, $1 \mathrm{Q} 858$ - Biens de première origine : clergé régulier : Cordeliers.

35. Arch. dép. d'Ille-et-Vilaine, 1 Q 855 - Biens de première origine : clergé régulier : Capucins.

36. Bibliothèque municipale de Rennes, Ms 562 - Catalogue des dépôts littéraires, op. cit. 
sent pas la totalité des possesseurs de livres visés par les confiscations, non plus, nous l'avons dit, que l'intégralité des bibliothèques concernées, mais de nombreux volumes confisqués ne sont jamais parvenus dans les dépôts littéraires. Certains ouvrages ont en effet échappé à la saisie proprement dite.

De plus, les bibliothèques demeurent dans les lieux en attendant leur transfert vers les dépôts littéraires, et les scellés constituent une faible protection contre les tentatives entreprises par les propriétaires en vue de récupérer leurs livres, ou bien contre les vols. De nombreuses disparitions de livres sont ainsi constatées, dans lesquelles sont impliqués certains commissaires. Lors de l'inventaire effectué chez La Bourdonnaye de Montluc, par exemple, plusieurs ouvrages ont disparu. Le commissaire est alors interrogé, pour savoir " s'il est vrai que lui commissaire, commis greffier ou scribe ou autres particuliers ont choisi dans la bibliothèque des ouvrages qu'ils ont vendus et achetés eux-mêmes et qu'ils ayent dit qu'il manquait des volumes ${ }^{37}$ ".

Certains livres seraient donc mis en vente malgré le décret datant du 10 octobre 1792 qui interdit cette pratique, et il est de fait que l'époque révolutionnaire connaît un important développement du commerce du livre d'occasion, alimenté par les saisies ${ }^{38}$. Des vols sont également commis lors du transfert des livres vers les dépôts littéraires. Il faut dire que l'opération est des plus hasardeuses : elle a lieu dans une grande confusion, aucun moyen n'étant prévu pour déplacer les livres, qui sont la plupart du temps entassés pêle-mêle dans des sacs ${ }^{39}$. Une fois les ouvrages entreposés dans les dépôts, ceux-ci, qui en renferment des milliers, suscitent à leur tour la convoitise. Des vols ont lieu à l'intérieur même des bâtiments, et malgré la présence d'une gardienne, la surveillance semble insuffisante : " On s'aperçut que la porte de la chambre où se trouvaient les livres des Bénédictins avait été enfoncée ${ }^{40}$. "

Enfin, les livres confisqués subissent de toute évidence un tri. Du moins constate-t-on que plusieurs des bibliothèques dont les inventaires figurent dans les dossiers des confiscations révolutionnaires ne sont curieusement pas présentes dans les dépôts littéraires. C'est le cas des livres des ursulines ${ }^{41}$, des religieuses de la Visitation de Saint-Melaine ${ }^{42}$ et des cordeliers ${ }^{43}$, qui, en toute logique, devraient pourtant avoir été transportés au dépôt littéraire. À titre

37. Arch. dép. d'Ille-et-Vilaine, 1 Q 1004 - Biens de deuxième origine : émigrés... Dossier individuel La Bourdonnaye de Montluc.

38. JOUON DES LONGRAIS, Frédéric, " Le commerce des vieux livres... ", art. cit., p. 13.

39. Arch. mun. de Rennes, 6 R 3 - Compte-rendu de l'état des dépôts littéraires aux administrateurs du département d'Ille-et-Vilaine, germinal an VII (mars-avril 1799).

40. Bibliothèque municipale de Rennes, Ms 560 - « Journal des travaux bibliographiques de Mainguy et Le Sage ", commissaires bibliographes du district de Rennes, an II-an IV.

41. Arch. dép. d'Ille-et-Vilaine, 1 Q 884-885 - Biens de première origine : clergé régulier : Grandes et Petites Ursulines.

42. Arch. dép. d'Ille-et-Vilaine, 1 Q 886 - Biens de première origine : clergé régulier : religieuses de la Visitation de Saint-Melaine.

43. Arch. dép. d'Ille-et-Vilaine, 1 Q 858 - Biens de première origine : clergé régulier : Cordeliers, op. cit. 
d'exemple, l'inventaire effectué chez les Grandes Ursulines porte seulement : " 23 in-octavo tous livres de piété, 360 in-12, sermons, livres de piété et de dévotion et plusieurs bouquins ${ }^{44}$. " On peut supposer que la composition de ces bibliothèques ressemblait fortement à d'autres bibliothèques de maisons religieuses inventoriées auparavant et que les commissaires, jugeant ces ouvrages inutiles, ont effectué un tri : cela expliquerait leur absence dans les dépôts littéraires. Les commissaires font d'ailleurs allusion à cette pratique :

" Nous avons également fait porter à l'évêché tous les livres de la bibliothèque pour les préposés faire triage de ceux qu'ils jugeront utiles à la bibliothèque nationale pour le surplus servir à l'emploi des cartouches ${ }^{45}$. "

Certains livres ont en effet servi à l'armée, le papier étant utilisé sous forme de cartouches ou de gargousses pour envelopper la poudre à canon ${ }^{46}$.

Retenons donc que les dépôts littéraires, destinés à former une bibliothèque publique, ne renferment pas la totalité des livres confisqués car certains ont été perdus ou volés, d'autres écartés après un travail de tri.

\section{Les dépôts littéraires}

\section{L'importance des dépôts}

Les dépôts littéraires rennais sont très importants quantitativement. Les confiscations révolutionnaires ont en effet concerné une masse considérable de livres. Si, nous l'avons dit, l'ampleur des saisies est difficile à évaluer, il est au moins possible de connaître le nombre global de livres qui composent les dépôts littéraires. Selon le catalogue établi par les deux commissaires Mainguy ${ }^{47}$ et Le Sage ${ }^{48}$, le nombre de volumes concernés

44. Arch. dép. d'Ille-et-Vilaine, 1 Q 884 - Biens de première origine : clergé régulier : Grandes Ursulines, op. cit.

45. Arch. dép. d'Ille-et-Vilaine, 1 Q 1091 - Biens de deuxième origine : membres du clergé. Dossier individuel Colin de la Biochais.

46. VARRY, Dominique (dir.), Histoire des bibliothèques françaises : les bibliothèques de la Révolution et du XIX $X^{e}$ siècle..., op. cit., p. 265.

47. Félix Alexis Mainguy naît à Rennes en 1747. Il entre au couvent des dominicains en 1763. Ordonné prêtre en 1770, il partage son temps entre l'enseignement et la prédication. Favorable aux idées nouvelles qui se répandent à la fin du xvIII ${ }^{\mathrm{e}}$ siècle, il est nommé commissaire pour la formation de la bibliothèque de Rennes en 1794. En 1796, il est bibliothécaire de l'école centrale, puis conservateur de la bibliothèque municipale en 1803. Il est également professeur entre 1804 et 1816, date à laquelle il devient aumônier du dépôt de mendicité. Il reste bibliothécaire jusqu'à sa mort, en 1818. Mainguy est assez représentatif des bibliothécaires de son époque, en majorité des ecclésiastiques et des hommes de lettres, le plus souvent favorables aux idées révolutionnaires. De plus, les fonctions de professeur et de bibliothécaire sont très fréquemment associées à cette époque. Considéré comme le fondateur de la bibliothèque municipale de Rennes, il a marqué l'histoire de l'établissement. Voir Toravel, Jean, " Félix Alexis Mainguy (17471818), dominicain, premier bibliothécaire de la ville de Rennes ", Bulletin et Mémoires de la Société archéologique du département d'Ille-et-Vilaine, t. 78, 1974, p. 95-119.

48. Pierre Michel Le Sage naît en 1760 Rennes. Son parcours est très proche de celui de Mainguy. Ancien dominicain, Le Sage est nommé commissaire bibliographe en 1794. 
est de 60000 en $1796^{49}$. Ce catalogue est un document fiable et précieux : parce qu'il répertorie tous les livres des différents dépôts, il constitue un témoin du premier état des collections destinées à former la bibliothèque publique de Rennes ${ }^{50}$.

Parmi les bibliothèques présentes dans les dépôts littéraires, six se détachent, qui comprennent entre 2000 et 7000 volumes. La plus importante est celle des grands carmes (7132 volumes). La deuxième est la bibliothèque des avocats (7113 volumes), puis vient la bibliothèque des Capucins (6 657 volumes). La bibliothèque du collectionneur de Robien contient quant à elle 4308 volumes. Enfin, la bibliothèque des jacobins est constituée de 3200 volumes et celle des bénédictins de 2009 volumes. Cinq bibliothèques contiennent environ mille volumes : celles des augustins et des minimes, du prêtre Macé et des avocats généraux Beaucourt et Bourgblanc. Les autres bibliothèques sont généralement composées de quelques centaines de volumes. Mais il en existe de bien plus petites : ainsi, cinq bibliothèques appartenant à des particuliers ou à des anonymes ne renferment que quelques dizaines de volumes. Les bibliothèques les plus importantes étaient donc la propriété de quelques riches parlementaires ou bien d'établissements religieux ${ }^{51}$.

\section{Leur contenu}

Dans son "Journal des travaux bibliographiques ", rédigé entre 1794 et 1796, Félix Mainguy relate ses travaux d'inventaire et nous permet ainsi d'entrevoir l'idée qu'il se faisait de l'intérêt des différents dépôts ${ }^{52}$. Mainguy souligne l'existence de très belles collections. Selon lui, la bibliothèque du parlementaire du Plessis de Grenedan est " superbe. Elle étale tout le luxe typographique. Elle est choisie en outre, mais il paraît qu'elle n'est point complète. La partie de jurisprudence ne s'y trouve point, non plus que l'histoire ${ }^{53}$ ".

Celle du prêtre Macé est " aussi nombreuse que choisie. On y trouve la fameuse polyglotte de Walton très estimée et la meilleure, beaucoup d'ouvrages grecs, beaucoup d'auteurs de médecine ${ }^{54}$ ". Mainguy évoque

\footnotetext{
Après le transfert de la bibliothèque à l'école centrale, Le Sage est maintenu dans ses fonctions de commissaire bibliographe. Il quitte cette activité pour celle de professeur en 1799. Réconcilié avec l'Église puisqu'il reprend ses fonctions sacerdotales dès 1801, il devient aumônier du lycée en 1808 et meurt en 1819. Voir TORAVEL, Jean, « Le Couvent des Dominicains de Rennes au xvIII siècle ", Bulletin et Mémoires de la Société archéologique du département d'Ille-et-Vilaine, t. 92, 1990, p. 181-243 et t. 93, 1991, p. 225-230.

49. Bibliothèque municipale de Rennes, Ms 562, Catalogue des dépôts littéraires, op. cit.

50. Ibidem.

51. L'importance de ce type de bibliothèques se retrouve dans la plupart des autres villes parlementaires, comme à Rouen, Dijon ou Bordeaux : voir Patrimoine des bibliothèques de France..., op. cit.

52. Bibliothèque municipale de Rennes, Ms 560 - « Journal des travaux bibliographiques...", op. cit.

53. Ibidem.

54. Ibid.
} 
aussi la bibliothèque des Grands Carmes : "Une collection considérable. Elle renferme de très bons ouvrages. Plusieurs touchent au berceau de la typographie. Beaucoup sont couverts en parchemin ${ }^{55}$. " Quant à celle de Robien, elle contient :

" des manuscrits précieux, des bréviaires gothiques historiés, ornés de figures, de vignettes, et sur velin, le Coran écrit en arabe sur papier gommé et très lisse de la Chine, une écorce d'arbre d'un pied et quelques pouces, sur laquelle sont tracés des deux côtés des caractères inconnus, largeur un pouce, un petit dictionnaire arabe et français sur papier gommé. Du reste la bibliothèque est choisie et sans doute à certains égards la meilleure bibliothèque de toutes celles que nous aurons à inventorier ${ }^{56}$ ".

Mainguy évoque aussi des bibliothèques qui, à l'inverse, présentent peu d'intérêt à ses yeux. La bibliothèque de l'évêque de Rennes, Bareau de Girac, est ainsi " presque uniquement composée d'ascétiques. Il n'y a aucun bon choix ${ }^{57}$ ". Les livres du vicaire de Gévezé, de la Noé, sont " peu nombreux et aucunement choisis ${ }^{58}$ ". Dans la bibliothèque de l'école de droit, Mainguy écrit n'avoir rien vu d'intéressant. Enfin, la bibliothèque des dames Budes se résume selon lui à des ouvrages d'ascétisme ${ }^{59}$. Ces jugements de Mainguy sont représentatifs de sa vision des choses et du regard qu'il porte sur les livres et la culture. À la fois ancien ecclésiastique et professeur, cet homme instruit, qui possède des connaissances bibliographiques, est capable de repérer les ouvrages rares, précieux et les manuscrits ou au contraire les livres obsolètes. Favorable aux idées nouvelles, il privilégie tout naturellement certains livres, notamment ceux de science, par rapport aux livres religieux, jugés inutiles pour la plupart.

On l'a deviné, le premier élément qui caractérise les dépôts littéraires de Rennes est le primat des collections religieuses. Avant les confiscations, celles-ci n'avaient d'autre objet que d'être utiles aux religieux (et dans une moindre mesure au public extérieur, dans le cas des quelques rares établissements où celui-ci était admis). Ces fonds provenant des bibliothèques ecclésiastiques et résultant d'une accumulation d'ouvrages de théologie effectuée sur plusieurs décennies, ils présentent une remarquable homogénéité. Ils sont surtout composés de Bibles, de sermons, de livres de piété et de dévotion, ainsi que d'histoire ecclésiastique. Lors de l'enquête du ministère de l'Intérieur, datant de 1799, qui pose cette question à propos des dépôts : "Quels genres d'ouvrages dominent dans ceux-ci? "; Mainguy répond : "Les ouvrages ecclésiastiques, la théologie, l'ascétisme ${ }^{60}$. " Mais

55. Ibid.

56. Ibid.

57. Ibid.

58. Ibid.

59. Arch. dép. d'llle-et-Vilaine, 1 Q 890 - Biens de première origine : clergé régulier : Dames Budes.

60. Arch. mun. de Rennes, 6 R 3 - Réponse aux questions du ministre de l'Intérieur sur l'état actuel des bibliothèques dans le département d'Ille-et-Vilaine, ventôse an VII (février-mars 1799). 
les dépôts littéraires contiennent également des bibliothèques d'émigrés, composées d'ouvrages plus modernes, au contenu souvent voisin, notamment lorsque leurs propriétaires exerçaient la même profession. En définitive, on constate que l'essentiel des collections confisquées est donc constitué de livres de théologie ${ }^{61}$, d'ouvrages incomplets ou en double. Cette conclusion est en tout cas celle du compte-rendu adressé par Mainguy aux administrateurs du département d'Ille-et-Vilaine en 1799 :

"Si on examine en détail les dépôts, la plupart étant ecclésiastiques, vous y trouvez beaucoup de théologie et d'ascétisme. D'autres appartenant à des hommes de loi, on y rencontre beaucoup d'auteurs de jurisprudence. Cette masse énorme de livres, réduite en raison de répétitions et d'insignifiance, perdra de son poids et de son volume. Toutes les communautés offrent les mêmes livres fondamentaux des bibliothèques. Que de Bibles! De commentaires! D'interprètes! De pères de l'Église! De conférences ecclésiastiques! D'auteurs de théologie! Et toujours les mêmes dans tous les dépôts! Même répétition dans la jurisprudence [...]. Cependant les deux bibliothèques, des avocats et de Robien, pourraient presque se réunir. Les parties qui ne se trouvent pas dans l'une, se trouvent dans l'autre [...]. Notre bibliothèque offre une immensité de livres, mais presque rien de moderne. Tout ce qui est nouveau, depuis vingt ou trente ans, dans les sciences et les arts, est presque étranger à nos dépôts littéraires (physique, chimie, astronomie.... ${ }^{62}$. "

Ainsi, malgré la quantité considérable de livres regroupés dans les dépôts, force est d'admettre que la majeure partie d'entre eux ne présente qu'un intérêt limité en vue de la constitution d'une bibliothèque publique.

\section{Au hasard des déménagements}

S'il s'avère difficile d'organiser les dépôts littéraires, c'est d'abord et avant tout en raison de l'inadaptation des locaux qui leur sont attribués. En effet, les bâtiments qui les abritent sont délabrés et la place manque : rien n'est prévu pour recevoir les livres. Les dépôts s'entassent donc par ordre de provenance. Il n'y a ni supports, ni rayonnages. En raison du manque d'étagères, les ouvrages sont simplement posés par terre, et les piles de livres jonchent le sol. La cohérence des diverses collections confisquées ne peut donc être respectée. De plus, les livres, qui doivent théoriquement être regroupés dans un dépôt commun, sont la plupart du temps dispersés; à peine sont-ils regroupés dans un endroit qu'il faut de nouveau les déplacer. Le travail de tri est à recommencer à chaque fois car les déménagements sont incessants ${ }^{63}$.

61. Selon le catalogue des dépôts littéraires rédigé à partir de 1794, et qui répertorie environ 60000 volumes, la catégorie théologie représente plus de 20000 volumes, c'est-àdire plus du tiers des collections (Bibliothèque municipale de Rennes, Ms 562 - Catalogue des dépôts littéraires, op. cit.).

62. Arch. mun. de Rennes, 6 R 3 - Compte-rendu de l'état des dépôts littéraires aux administrateurs du département d'Ille-et-Vilaine, germinal an VII (mars-avril 1799).

63. L'histoire de ces déménagements a été retracée dans trois principales études : FERrIEU, Xavier, "La constitution des fonds de la bibliothèque municipale de Rennes ", 


\section{Au couvent de la Visitation}

Les livres saisis sont tout d'abord regroupés en 1792 dans une quarantaine de dépôts provisoires, aussi bien chez des particuliers que dans des locaux officiels. Puis, en 1793, tous les ouvrages et les objets d'art provenant des confiscations sont entreposés dans un dépôt commun installé dans la chapelle du couvent de la Visitation. Précisons tout de suite que si tous ces objets et ces livres sont au départ confondus, ils se sépareront plus tard pour former une bibliothèque et trois musées ${ }^{64}$. Le dépôt commun demeure pêle-mêle plusieurs mois au couvent de la Visitation. Mais en novembre 1793, après la prise de Fougères, les troupes républicaines, pressées par l'armée vendéenne, sont contraintes de se replier sur Rennes. Le couvent de la Visitation est alors transformé en hôpital pour recueillir les blessés, et certains livres sont découpés afin de servir à la fabrication de pansements :

"Les malades étaient au milieu des livres. Les chirurgiens se servaient des livres hébreux pour les emplâtres, et la forme sphérique se trouve encore sur ces ouvrages précieux et antiques ${ }^{65}$."

Comme il n'est plus possible de laisser le dépôt littéraire à cet endroit, on décide de le transférer non loin de là, au couvent des carmélites.

\section{Les tribulations de la bibliothèque de district : du couvent des Carmélites à l'évêché}

C'est peu après, en application du décret du 8 pluviôse an II (27 janvier 1794), que le dépôt se transforme en bibliothèque de district, et un peu plus tard encore, en mars 1794, que, nous l'avons vu, Mainguy et Le Sage sont nommés par le district " commissaires bibliographes " afin d'inventorier les livres dans le cadre du projet de «Bibliographie universelle ${ }^{66}$ ». Ceux-ci

in Charpiana. Mélanges offerts par ses amis à Jacques Charpy, Rennes, Fédération des sociétés savantes de Bretagne, 1991, p. 69-70; HoulBERT, Constant, Le Musée d'Histoire naturelle de la ville de Rennes, guide historique et descriptif, origines et accroissement des principales collections (1794-1928), Rennes, Oberthur, 1933, p. 12-64; ToRAVEL, Jean, "Félix Alexis Mainguy (1747-1818)...", art. cit., p. 110-118.

64. Les confiscations révolutionnaires sont en effet à l'origine de la bibliothèque municipale de Rennes, mais aussi du musée des beaux-arts, du musée d'archéologie et du musée d'histoire naturelle.

65. Arch. mun. de Rennes, 6 R 3 - Rapport de Mainguy au maire en 1813.

66. D’Ormesson, bibliothécaire du roi, présente son projet de «Bibliographie universelle " en 1790. Il a pour objectif d'inventorier l'ensemble des ouvrages contenus dans les dépôts littéraires, afin de constituer le premier catalogue collectif à l'échelon national et de rendre ces livres accessibles au plus grand nombre. La réalisation des catalogues repose sur l'utilisation de cartes à jouer. Le verso de ces cartes, vierge de toute inscription et de tout dessin, sert à la rédaction de notices bibliographiques. Ces cartes sont expédiées par les districts à Paris, où des commis spécialisés procèdent à leur dépouillement sur des feuilles récapitulatives. À ce sujet, voir les travaux de RIBERETTE, Pierre, Les Bibliothèques françaises pendant la Révolution (1789-1795) : recherches sur un essai de catalogue collectif, Paris, Bibliothèque nationale, 1970, 157 p. Les commissaires 
trouvent vite le local des Carmélites insuffisant; ils se plaignent de manquer de place pour effectuer leurs travaux d'inventaire. Ils adressent alors une demande aux administrateurs du district pour obtenir que le dépôt soit transféré à l'évêché. Le 2 septembre 1794, le district donne son accord, demandant que le transport des livres se fasse immédiatement ${ }^{67}$. Le transfert de cette immense quantité de livres et d'objets commence à l'automne, et de nombreuses opérations de transport se succèdent. L'évêché devient ainsi le dépôt commun de la bibliothèque et des musées (seule la bibliothèque des avocats, confisquée en 1794, reste à sa place au présidial et demeure séparée des autres dépôts littéraires). Mais même à l'intérieur de ce bâtiment, les déménagements se poursuivent encore. Les livres sont à peine rangés au premier étage que, le $1^{\text {er }}$ février 1795, le représentant Boursault ordonne de transporter les différents dépôts littéraires au deuxième étage ${ }^{68}$. Puis, le 14 juillet 1795, deux autres représentants du peuple, voulant occuper l'appartement dans lequel sont placés les livres des Minimes, demandent que ceuxci soient déplacés. Enfin, cinq jours plus tard, le représentant Mathieu, qui vient visiter le muséum, exige le transfert du dépôt littéraire des Bénédictins car il dit avoir besoin du cabinet avec cheminée pour son secrétaire ${ }^{69}$. En raison du manque de place à l'évêché, une partie des livres est alors transférée juste en face, à l'abbaye de Saint-Melaine. Malheureusement, un incendie survient en 1796, et les livres qui s'y trouvaient sont brûlés ${ }^{70}$.

Au départ, la bibliothèque de district ne fonctionne pas vraiment. C'est qu'il n'est évidemment pas possible de la rendre accessible au public tant que les travaux bibliographiques ne sont pas achevés. La situation change à la fin de l'année 1795 : en novembre, la bibliothèque des avocats, située au présidial, ouvre ses portes au public les jours pairs, celle située à l'évêché les jours impairs ${ }^{71}$. Mais au début de l'année 1796, l'évêché devant être

\footnotetext{
Mainguy et Le Sage confectionnent un catalogue destiné à rester sur place et rédigent également un catalogue sur des cartes à jouer destiné à être envoyé à Paris au bureau de la Bibliographie (Bibliothèque municipale de Rennes, Ms 562 - Catalogue des dépôts littéraires). Le district de Rennes aurait envoyé au bureau de la Bibliographie 42 inventaires, et 14329 cartes. Voir à ce sujet VARRY, Dominique (dir.), Histoire des bibliothèques françaises : les bibliothèques de la Révolution et du XIX siècle..., op. cit., p. 22-23 (" Tableau 3, Commisssion temporaire des arts : relevé des catalogues bibliographiques faits sur cartes et envoyés par les districts au bureau de la Bibliographie générale, [An III] "). Ce chiffre de 14329 est assez élevé, car selon ce tableau la moitié des districts a envoyé moins de 10000 cartes. Les deux commissaires Mainguy et Le Sage ont inventorié la majorité des dépôts littéraires à la fin du mois d'octobre 1795, peu avant l'abandon du projet de "Bibliographie universelle ", qui se solde par un échec en 1796.

67. Arch. mun. de Rennes, 6 R 3 - Séance de l'administration du district du 16 fructidor an II (2 septembre 1794).

68. Arch. mun. de Rennes, 6R 3 - Compte-rendu de l'état des dépôts littéraires aux administrateurs du département d'Ille-et-Vilaine, germinal an VII (mars-avril 1799), op. cit.

69. MallLet, Dominique, Origines de la bibliothèque municipale de Rennes, Rennes, Vannier, p. 22.

70. Arch. mun. de Rennes, 6 R 3 - Rapport de Mainguy au maire en 1813.

71. HoulBert, Constant, Le Musée d'Histoire naturelle de la ville de Rennes..., op. cit., p. 43.
} 
occupé par le général Simon, il faut de nouveau évacuer l'ensemble des livres.

\section{Transfert à l'école centrale}

À cette date, les districts sont déjà supprimés depuis plusieurs mois et des écoles centrales ont vu le jour, depuis précisément la loi Lakanal du 7 ventôse an III (25 février 1795). Cette loi prévoit l'existence d'une école centrale par département et stipule que chacune d'elle doit posséder une bibliothèque publique ${ }^{72}$. Elle est complétée par la circulaire du 5 mai 1796 relative à la mise en place de ces bibliothèques, qui vient véritablement lier le sort des dépôts littéraires à celui des écoles centrales. C'est donc tout naturellement vers l'école centrale de Rennes que les livres conservés jusque là à l'évêché sont dirigés en 1796. Et c'est alors, notons-le, que la nouvelle bibliothèque se sépare des musées et devient indépendante. Installée dans les locaux de l'ancien collège, l'école centrale ouvre ses portes à la fin du mois de septembre 1796. Mainguy, l'un des deux anciens commissaires bibliographes de la bibliothèque de district, en devient officiellement le bibliothécaire le 21 du même mois ${ }^{73}$. Elle est ouverte aux élèves, aux enseignants, mais également au public extérieur. Ainsi joue-telle un double rôle de bibliothèque scolaire et de bibliothèque publique, ce qui explique qu'elle ne doit pas seulement couvrir les matières enseignées à l'école, mais également conserver les collections générales intéressant d'autres domaines.

\section{La naissance de la bibliothèque municipale et son installation à l'hôtel de ville}

La loi du $1^{\mathrm{er}}$ mai 1802 supprime les écoles centrales et les remplace par les lycées, condamnant ipso facto les bibliothèques des écoles centrales à disparaître. Les livres subissent donc un nouveau déménagement, qui dure quinze jours ${ }^{74}$. Les collections sont finalement entreposées à l'hôtel de ville, au-dessus de l'ancien présidial. Elles rejoignent ainsi les livres de la bibliothèque des avocats, qui était restée dans son ancien local. La suppression des écoles centrales est suivie par le décret du 8 pluviôse an XI (28 janvier 1803), qui fixe le sort de leurs bibliothèques. Celles-ci sont " mises à la disposition et sous la surveillance de la municipalité ". En somme, le gouvernement se décharge de leur entretien sans en abandonner la propriété. Ce décret constitue l'acte de naissance officielle des bibliothèques municipales en France. Le 20 ventôse an XI (11 mars 1803),

72. CANARD, Alain et YSNEL, Frédéric, "Un Musée d'histoire naturelle à Rennes : un renouveau " Bulletin et Mémoires de la Société archéologique du département d'Ille-et-Vilaine, t. 102, 2003, p. 285.

73. Arc. mun. de Rennes, 6 R 1 - Extrait des registres du jury central d'instruction publique, $5^{\mathrm{e}}$ jour complémentaire an IV (21 septembre 1796).

74. MaIllet, Dominique, Origines de la bibliothèque municipale de Rennes, op. cit., p. 26. 
le préfet prend un arrêté qui crée la bibliothèque municipale de Rennes. Mainguy est nommé conservateur de l'établissement le lendemain de sa création, le 12 mars $1803^{75}$.

En définitive, après de nombreux déménagements, et après avoir occupé différents bâtiments (le couvent de la Visitation, le couvent des Carmélites, l'évêché, l'abbaye Saint-Melaine, l'école centrale et enfin l'hôtel de ville), la bibliothèque de Rennes, constituée à partir des livres contenus dans les dépôts littéraires, est devenue bibliothèque municipale une dizaine d'années plus tard, après avoir connu divers statuts (simple dépôt littéraire, bibliothèque de district, bibliothèque de l'école centrale et enfin bibliothèque municipale).

Au cours de ces années 1789-1803, l'histoire de la bibliothèque municipale de Rennes présente de multiples similitudes avec celle des autres bibliothèques françaises. À Rennes comme ailleurs, la mise en place de la bibliothèque s'est heurtée à de nombreux obstacles tels que l'inadaptation des locaux, les conditions déplorables de conservation des ouvrages, leurs nombreux déménagements, les vols dont ils ont été l'objet, la dispersion des fonds, la difficile organisation des collections, qui a nécessité un énorme travail de tri et de catalogage, ou encore le manque de moyens financiers. De même, constate-t-on que les collections confisquées sont majoritairement composées d'ouvrages de théologie provenant des anciennes communautés religieuses, que l'impératif de conservation des fonds l'emporte encore sur le souci de les rendre accessibles au public, enfin que les collections sont avant tout fréquentées par les élites locales : toutes choses qui autorisent à conclure que la volonté des révolutionnaires de répandre les Lumières a échoué sur le court terme. En somme, ce que montre l'exemple rennais, après d'autres, c'est que la Révolution, si elle a hautement proclamé l'idéal de démocratisation culturelle, n'a pas su le réaliser concrètement.

Ce constat doit être cependant doublement tempéré. En premier lieu, le bilan de la Révolution n'est pas entièrement négatif. S'il est vrai que les réalisations n'ont pas été à la hauteur des ambitions initiales, il n'est pas moins vrai que cette période a été fondatrice dans l'histoire des bibliothèques françaises, d'abord parce qu'elle leur a fourni un cadre institutionnel appelé à durer, ensuite parce qu'elle les a dotées de fonds volumineux. Rappelons que les confiscations révolutionnaires sont à l'origine des fonds anciens de la plupart des bibliothèques municipales classées. En France, cinquantequatre bibliothèques exactement, dont celle de Rennes, conservent des collections patrimoniales appartenant à l'État. Ainsi voit-on que l'histoire ultérieure des bibliothèques, au XIX ${ }^{\mathrm{e}}$ siècle notamment, doit malgré tout beaucoup aux innovations révolutionnaires ${ }^{76}$.

75. Arch. mun. de Rennes, 2 D 1 - Arrêté du maire, 21 ventôse an XI (12 mars 1803), p. 102.

76. Rioux, Jean-Pierre et SiRINELLI, Jean-François (dir.), Histoire culturelle de la France, vol. 3, op. cit., p. 150. 
En second lieu, l'exemple rennais montre que ces innovations, quelque convergentes qu'elles aient été d'une ville à l'autre, se singularisent localement. En effet, même si les déménagements répétés d'un dépôt littéraire à un autre ont été courants et si la plupart des bibliothèques de province sont passées par les mêmes étapes - dépôts littéraires, bibliothèque de district, de l'école centrale et enfin bibliothèque municipale -, celle de Rennes a bel et bien sa propre histoire. Son originalité tient à un jeu de contraintes spécifiques qui a abouti à la scission durable de ses collections : d'un côté le dépôt de la bibliothèque des avocats, demeuré au-dessus de l'ancien présidial et qui a ainsi conservé son emplacement d'origine, de l'autre le reste des dépôts, successivement regroupés dans la chapelle du couvent de la Visitation, aux Carmélites, à l'évêché et enfin à l'école centrale. On a vu que les deux bibliothèques n'ont fusionné qu'en 1803, lorsque la bibliothèque municipale a été installée à l'hôtel de ville. On peut à présent noter que l'idée répandue localement selon laquelle la bibliothèque des avocats est l'ancêtre de la bibliothèque municipale est largement erronée. La comparaison avec d'autres villes permet de bien faire ressortir la particularité du cas rennais. Nous pouvons citer Bordeaux et Toulouse, deux villes bénéficiant tout comme Rennes de la présence d'un parlement. À Bordeaux, la bibliothèque de l'Académie, ouverte en 1740, confisquée au moment de la Révolution, a constitué le noyau de la future bibliothèque municipale, qui est demeurée dans le même lieu ${ }^{77}$. Le cas de Toulouse est totalement différent : la ville possédait deux bibliothèques ouvertes au public à la fin du $\mathrm{XVIII}^{\mathrm{e}}$ siècle : la bibliothèque du clergé et celle du collège royal - l'ancienne bibliothèque du collège des Jésuites. À la Révolution, tandis que la bibliothèque du clergé est restée dans son local, la bibliothèque du collège royal - devenu national puis école centrale - s'est accrue des dépôts provenant des confiscations et est devenue bibliothèque municipale en 1803. Les deux bibliothèques sont donc demeurées séparées, et ont ouvert au public à tour de rôle. Leur fusion ne se fera qu'en $1866^{78}$. Si le cas de Toulouse présente des similitudes avec le cas rennais, attendu que dans les années suivant la Révolution, les deux villes ont connu l'existence de deux bibliothèques fonctionnant simultanément, l'histoire des bibliothèques municipales de ces deux villes n'est cependant pas identique, tant s'en faut.

Il faut dire que l'action de Mainguy a fortement pesé sur les premières années d'existence de la bibliothèque municipale de Rennes et marqué d'une empreinte propre son développement ultérieur. Mainguy poursuivra son travail après 1803. Il se consacrera tout particulièrement à l'organisation des collections. Malgré des difficultés financières, liées au fait que la bibliothèque ne bénéficie au départ d'aucun budget, Mainguy effectuera un important travail de tri, réalisant des acquisitions, effectuant des ventes, procédant à des dons d'ouvrages. Des 60000 volumes provenant des confis-

77. "Bibliothèque municipale de Bordeaux ", dans : Patrimoine des bibliothèques de France..., op. cit., vol. 7 (Aquitaine, Languedoc-Roussillon, Midi-Pyrénées), p. 66.

78. "Bibliothèque municipale de Toulouse ", ibid., p. 238. 
cations révolutionnaires, il n'en restera plus que 30000 à sa mort, en 1818 . Cette importante diminution des fonds a surtout concerné les livres de théologie, mais aussi de jurisprudence, exclus des collections au profit d'ouvrages plus modernes, notamment dans les domaines des sciences et des $\operatorname{arts}^{79}$. La bibliothèque élargira parallèlement ses horaires d'ouverture $^{80}$; elle touchera, au bout d'une dizaine d'années, 150 à 200 lecteurs réguliers ${ }^{81}$. Pour autant, à cette date, l'idéal de bibliothèque publique est encore loin d'être réalisé : l'heure est toujours à la conservation des collections, bien plus qu'à leur mise à disposition du public, et la poignée de lecteurs qui fréquentent l'établissement - principalement des avocats et des étudiants - appartiennent à l'élite intellectuelle rennaise ${ }^{82}$.

79. Ainsi, on constate qu'en 1818, les livres de théologie, qui représentaient plus d'un tiers des collections en 1796, ne sont plus qu'au nombre de 2700 environ, 20000 volumes ayant été donnés au séminaire. La catégorie jurisprudence, très importante en 1796 , a aussi énormément diminué puisqu'elle ne contient plus que 2800 volumes en 1818. Les catégories histoire et belles-lettres ont conservé leur importance, avec respectivement 5600 et 4500 volumes environ. Quant à la catégorie sciences et arts, pratiquement inexistante en 1796, elle compte plus de 3000 volumes en 1818. Cette évolution des collections est visible à la lecture de deux catalogues, celui des dépôts littéraires élaboré entre 1794 et 1796 et celui rédigé entre 1803 et 1818 (bibliothèque municipale de Rennes, Ms 562 Catalogue des dépôts littéraires et Ms 571-580 - Catalogues de la bibliothèque de Rennes, 1803).

80. Arch. mun. de Rennes, 2 D 4 - Arrêté du maire, p. 65 (règlement de la bibliothèque municipale de Rennes du 10 octobre 1810) et 2 D 5 - Arrêté du maire, p. 21-22 (règlement du $1^{\mathrm{er}}$ octobre 1811).

81. Arch. mun. de Rennes, 6R 1 - Lettre de Mainguy au préfet, 22 janvier 1813.

82. Arch. mun. de Rennes, 6R 1 - Lettre de Mainguy au préfet, 22 janvier 1813. 


\section{RÉSUMÉ}

La Révolution constitue une période fondatrice dans l'histoire des bibliothèques françaises. L'étude du cas rennais et de l'application locale de mesures décidées au niveau national, permet une approche concrète de la naissance d'une bibliothèque municipale de province. Celle de Rennes a sa propre histoire et ses particularités, tout en étant passée par les mêmes étapes que la plupart des autres bibliothèques. Lors des confiscations révolutionnaires, entre 1789 et $\mathbf{1 7 9 3}$, les bibliothèques privées rennaises sont saisies et les livres font l'objet d'inventaires, afin de répertorier les collections confisquées. Puis, les ouvrages sont regroupés dans des dépôts littéraires, où ils subissent de très mauvaises conditions de conservation. Ces dépôts renferment une quantité importante de volumes, qui sont pour la plupart des livres de théologie provenant d'établissements religieux, mais aussi de nombreux ouvrages incomplets ou en double. Leur intérêt semble donc limité pour la constitution d'une bibliothèque publique. La mise en place de celle-ci se heurte également à d'autres obstacles. Ainsi, les collections subissent de nombreux déménagements et sont transférées dans différents bâtiments successifs. La bibliothèque connaît divers statuts (simple dépôt littéraire, bibliothèque de district, puis bibliothèque de l'école centrale) avant d'être installée à l'hôtel de ville et de devenir bibliothèque municipale en 1803 .

\section{ABSTRACT}

The French Revolution is a foundation time in the history of French libraries. The study of Rennes and of the local application of measures decided at national level allows a concrete approach to the birth of a provincial library. That of Rennes has its own history and characteristics, while having passed through the same steps as most other libraries. During the revolutionary confiscations, between 1789 and 1793, the private libraries of Rennes are seized and the books are inventoried, in order to identify the confiscated collections. Then, the books are grouped together into literary depots, where they suffer from bad storage conditions. These depots contain a lot of volumes, mostly books of theology from religious institutions, but also many incomplete works, or several copies of the same book. So, their interest seems limited for the formation of a public library. The setting-up of it also faces other obstacles. Indeed, the collections are subjected to frequent moves and are transferred to successive different buildings. The library knows several statuses (mere literary depot, district library, then central school library) before being installed in the Town Hall and becoming a public library in 1803. 